\title{
Monte Carlo simulations of corrosion inhibition of copper by two Schiff bases
}

\author{
Lei Guo ${ }^{a,}{ }^{*}$, Xiaolei Ren, Yang Zhou, Shenying Xu, Yulong Gong \\ School of Chemistry and Chemical Engineering, Chongqing University, Chongqing 400044, China \\ acqglei@163.com
}

Keywords: Schiff base, copper, corrosion inhibitor, Monte Carlo simulation.

\begin{abstract}
Atomistic modelling and simulations are becoming increasingly important in the field of corrosion inhibition. In this work, Monte Carlo simulations technique incorporating molecular mechanics and molecular dynamics was used to simulate the adsorption of two Schiff base derivatives, namely 4-(4-aminostyryl)-N,N-dimethyl aniline (AND) and 2-((4-(4-(dimethyl amino) story) phenyl imino) methyl) (DSM), on the copper surface. Our results indicate that both compounds are potential corrosion inhibitors for copper, and the inhibition efficiency of DSM is better than AND.
\end{abstract}

\section{Introduction}

Corrosion of copper is a major concern in the petrochemical industry due to the destructive attack of iron substrates by chemical and electrochemical reactions. In order to eliminate this undesirable destructive effect, various solutions have been put to use, such as the adoption of corrosion inhibitor, electrochemical protection, metal and non-metallic coating protective technology. Among them, the employment of corrosion inhibitors has been proved to be one of the most practical and efficient method [1]. A corrosion inhibitor is a chemical substance which, when added in small concentrations to an environment, minimizes or prevents corrosion.

Most well-known corrosion inhibitors are organic compounds having polar groups containing N, S, and/or $\mathrm{O}$ atoms and heterocyclic compounds with polar functional groups and conjugated double bonds [2]. Theoretical chemistry has been used recently to explain the mechanism of corrosion inhibition. As validated by Refs. [3, 4], the Metropolis Monte Carlo simulation has been an effective tool to study the interaction of inhibitors with the metal surface.

The aim of the present work is to study the influence of molecular structure on the inhibition of copper corrosion using chemical Monte Carlo simulation, and to explore the adsorption mechanism of two Schiff base compounds (see Fig. 1, i.e., AND, DSM) on Cu(111) surface.

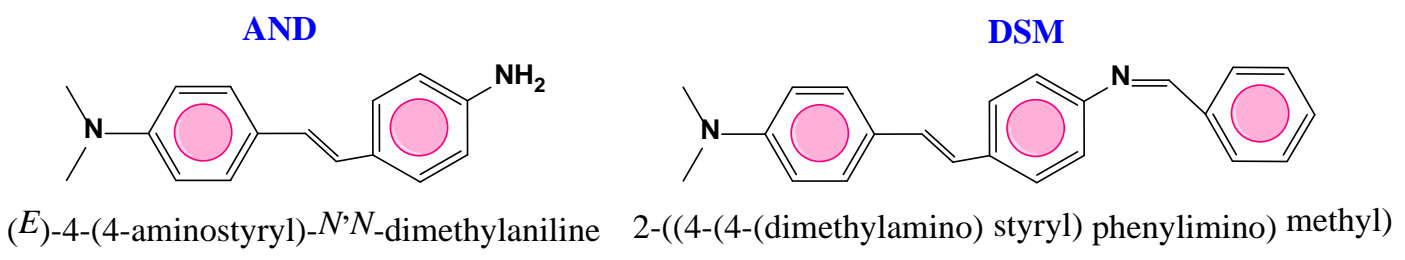

Fig. 1 the molecular structure of AND, DSM

\section{Computational details}

The interaction between a single inhibitor molecule and the copper surface was investigated by performing Monte Carlo simulations using the adsorption locator module of the Materials Studio software from Acers Inc. The surface $\mathrm{Cu}$ (111) was chosen to simulate the adsorption process. The calculations were carried out, using the COMPASS force field [5], in a simulation box (25.5 $\AA 25.5$ $\AA \times 50.4 \AA$ ) with periodic boundary conditions in order to simulate a representative part of an interface devoid of any arbitrary boundary effects. The Ewald \& group method (Ewald accuracy: 1.0 
$\times 10^{-5} \mathrm{kcal} / \mathrm{mol}$ ) was applied for the electrostatic interaction, and atom based method (cutoff distance: $1.85 \mathrm{~nm}$ ) for the van der Waals interaction.

A low-energy adsorption site is identified by carrying out a Monte Carlo search of the configurationally space of the substrate-adsorb ate system as the temperature is slowly decreased (simulated annealing). This process is repeated to identify further local energy minima. During the course of the simulation, adsorb ate molecules are randomly rotated and translated around the substrate. The configuration that results from one of these steps is accepted or rejected according to the selection rules of the Metropolis Monte Carlo method [6].

\section{Results and Discussion}

The total energy, average total energy, Vander Waals energy, electrostatic energy and intramolecular energy for inhibitor/Cu (111) surface are calculated by optimizing the whole system and presented in Fig. 2. The most stable adsorption configurations are shown in Fig. 3, it can be noticed that two inhibitors adsorbed nearly parallel (side view) to the copper surface through donation of $\pi$ electrons of the benzene rings and the lone pair of the hetero-atoms to the copper metal. This configuration is similar to our previous research about another corrosion inhibitor (3-indolebutyric acid) [7].
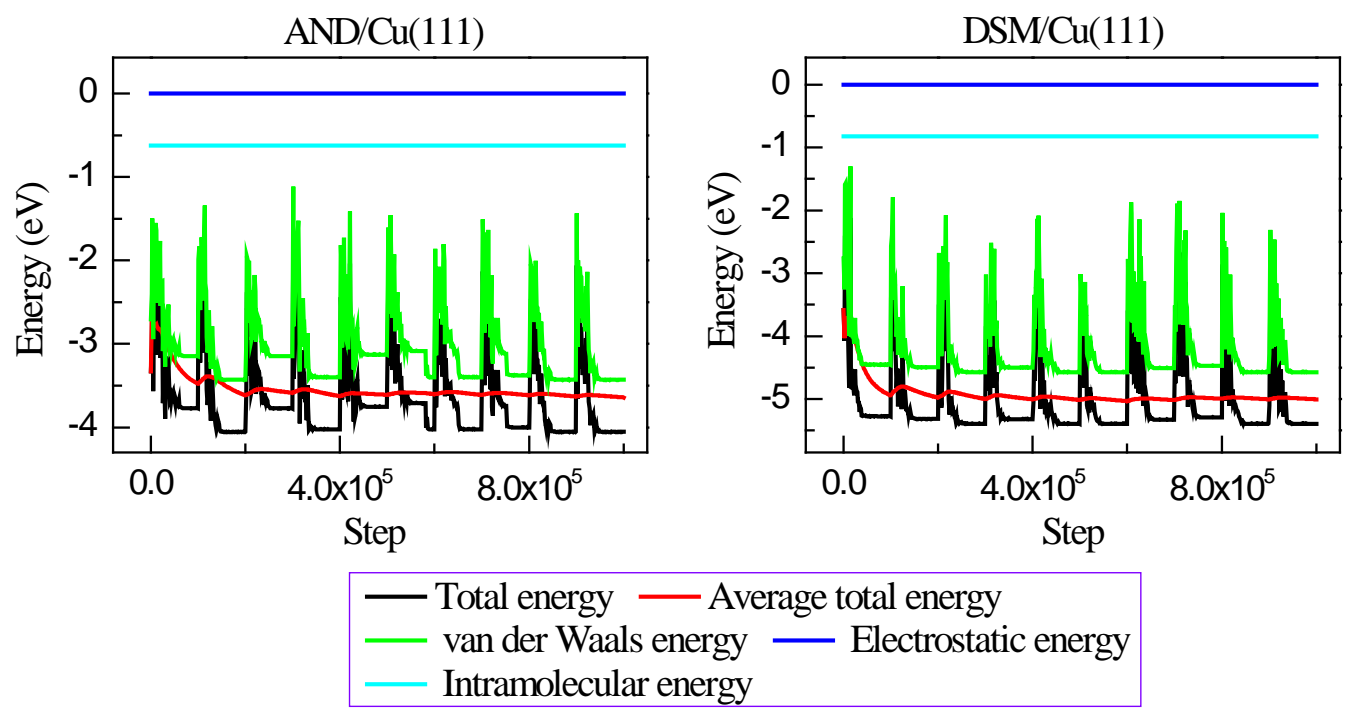

Fig. 2 Total energy distribution for the inhibitor/Cu (111) system during the energy optimization process
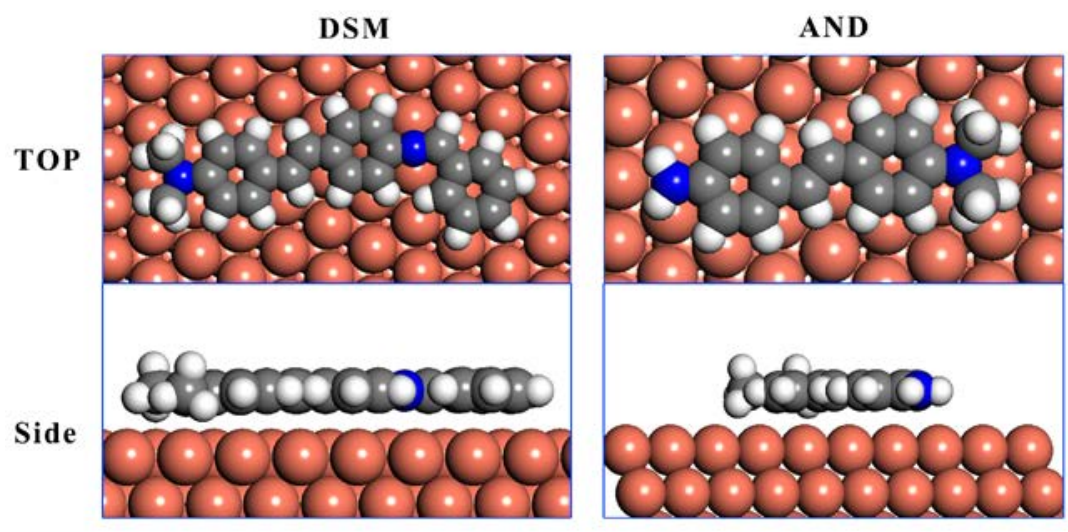

Fig. 3 the most stable configurations for adsorption of DSM/AND molecules on Cu (111) surface

Several outputs and descriptors calculated by the Monte Carlo simulation are presented in Table 1. The parameters include total energy of the substrate-adsorb ate configuration, which is defined as the sum of the energies of adsorb ate components, the rigid adsorption energy, and the deformation energy. The substrate energy (namely, $\mathrm{Cu}$ (111) surface) is taken as zero. Besides, adsorption energy 
reports the energy released (or required) when the relaxed adsorb ate component was adsorbed on the substrate. The adsorption energy is defined as the sum of the rigid adsorption energy and the deformation energy for adsorb ate component. The rigid adsorption energy reports the energy released (or required) when the unrelaxed adsorb ate component (before the geometry optimization step) was adsorbed on the substrate. The deformation energy reports the energy released when the adsorbed adsorb ate component was relaxed on the substrate surface. Table 1 also gives $\left(\mathrm{d} E_{\mathrm{ad}} / \mathrm{d} N_{\mathrm{i}}\right)$, which reports the energy of substrate-adsorb ate configurations where one of adsorb ate component has been removed.

Table 1 Outputs and descriptors calculated by the Mont Carlo simulation for adsorption of AND/DSM on Cu (111) (in eV)

\begin{tabular}{cccccc}
\hline $\begin{array}{c}\text { inhibito } \\
\text { r }\end{array}$ & $\begin{array}{c}\text { total } \\
\text { energy }\end{array}$ & $\begin{array}{c}\text { adsorption } \\
\text { energy }\end{array}$ & $\begin{array}{c}\text { rigid adsorption } \\
\text { energy }\end{array}$ & $\begin{array}{c}\text { deformation } \\
\text { energy }\end{array}$ & dEad/dNi \\
\hline AND & -4.28 & -3.65 & -3.69 & 0.03 & -3.65 \\
DSM & -5.72 & -4.90 & -4.94 & 0.04 & -4.90 \\
\hline
\end{tabular}

As can be seen from Table 1, the calculated adsorption energy values of the adsorption systems were -3.65 and $-4.90 \mathrm{eV}$ for AND DSM, respectively. Both values of adsorption energies are negative, which means that the adsorption could occur spontaneously. The larger negative values of interaction energy can be attributed to the strong adsorption between the inhibitor molecules and the copper surface. It is obvious that DSM gives a more negative adsorption energy, which means that adsorption of DSM on Cu (111) surface is stronger than AND. High value of adsorption energy indicates that DSM molecule is possible efficient inhibitor.
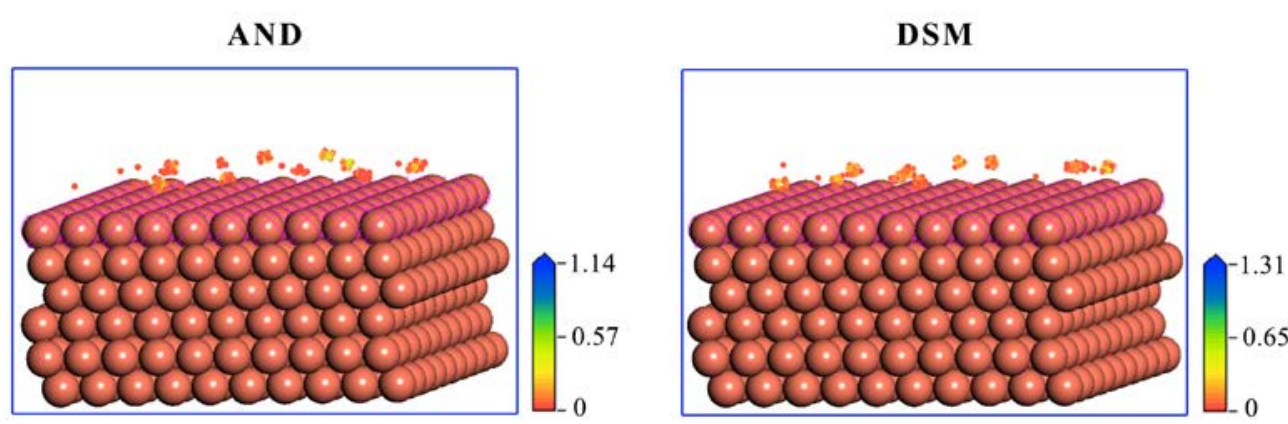

Fig. 4 The density field for adsorption of AND/DSM on Cu (111) substrate

The adsorption density of AND/DSM on Cu (111) substrate has been displayed in Fig. 4. To some extent, it is not hard to see that DSM has a higher density than AND on the Cu (111) surface.

The dissolution of copper in acidic solution proceeded according to the following steps:

$$
\begin{aligned}
& \mathrm{Cu}-e^{-} \rightarrow \mathrm{Cu}(\mathrm{I})_{\text {ads }} \quad \text { (Fast) } \\
& \mathrm{Cu}(\mathrm{I})_{\text {ads }}-e^{-} \rightarrow \mathrm{Cu}(\text { II) (Slow) }
\end{aligned}
$$

Where $\mathrm{Cu}(\mathrm{I})_{\text {ads }}$ is an adsorbed species at the copper surface and does not diffuse into the bulk solution. The cathode reduction of oxygen can be expressed as follow:

$$
\mathrm{O}_{2}+4 \mathrm{H}^{+}+4 e^{-} \rightarrow 2 \mathrm{H}_{2} \mathrm{O}
$$

A mechanism has been proposed to explain the inhibiting efficiency recorded for the inhibitors of interest, designated as INH. It attributes the phenomenon to the formation of an adsorbed layer of $\mathrm{INH}$, i.e.

$$
\mathrm{Cu}_{(\mathrm{s})}+\mathrm{INH} \rightarrow \mathrm{Cu}: \mathrm{INH}_{(\mathrm{ads})}
$$

Where Cu: INH (ads) refers to INH adsorbed on the copper surface. Thus, as indicated in Fig. 5, the studied inhibitor is likely to adsorb on the copper surface to form stable organic adsorption layers, and can be seen as a barrier to protect copper from corrosion. 


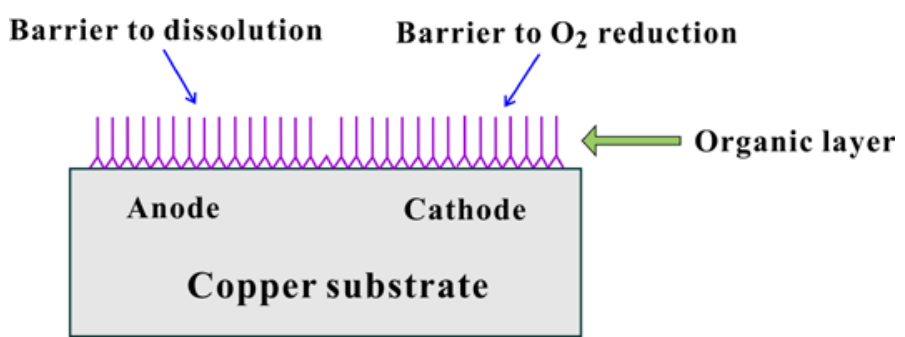

Fig. 5 Proposed scheme for the adsorption of inhibitors on the copper surface in acid medium

\section{Summary}

Understanding the adsorption phenomena is very important in corrosion problems. Theoretical studies help to find the most stable adsorption sites for a broad range of materials. This information can help to gain further insight about the corrosion system, such as the most likely point of attack for corrosion on a surface, the most stable site for inhibitor adsorption, and the adsorption energy of the adsorbed layer.

The simulation suggests that AND/DSM molecules adsorb on $\mathrm{Cu}$ (111) surface in a planar manner with a high negative adsorption energy. Yet, DSM has better corrosion inhibition performance than AND.

\section{Acknowledgements}

This research was supported by Natural Science Foundation of China (No.21376282), and Chongqing Innovation Fund for Graduate Students (No.CYB14019).

\section{References}

[1] B.E.A. Rani, B.B.J. Basu, Green inhibitors for corrosion protection of metals and alloys: an overview, Int. J. Corros. 2012 (2012) 1-15.

[2] D. Kesavan, M. Gopiraman, N. Sulochana, Green inhibitors for corrosion of metals: a review, Che. Sci. Rev. Lett. 1 (2012) 1-8.

[3] K.F. Khaled, Monte Carlo simulations of corrosion inhibition of mild steel in $0.5 \mathrm{M}$ sulphuric acid by some green corrosion inhibitors, J. Solid State Electrochemist. 13 (2009) 1743-1756.

[4] S.R. Jale, M. Bulow, F.R. Fitch, et al., Monte Carlo simulation of sorption equilibria for nitrogen and oxygen on LiLSX zeolite, J. Phys. Chem. B 104 (2000) 5272-5280.

[5] H. Sun, P. Ren, J.R. Fried, The COMPASS force field: parameterization and validation for phosphazenes, Compute. Theory. Polyp. Sci. 8 (1998) 229-246.

[6] R.S. Dumont, A Metropolis Monte Carlo method for computing micro-canonical statistical rate constants, J. Compute. Chem. 12 (1991) 391-401.

[7] J.H. Tan, L. Guo, T.M. Lv, et al., Experimental and computational evaluation of 3-indolebutyric acid as a corrosion inhibitor for mild steel in sulfuric acid solution, Int. J. Electrochemist. Sci., 10 (2015) 823-837. 